REVISTA MATEMÁTICA de la

Universidad Complutense de Madrid

Volumen 9, número 2: 1996

http://dx.doi.org/10.5209/rev_REMA.1996.v9.n2.17571

\title{
Orthonormal bases for spaces of continuous and continuously differentiable functions defined on a subset of $Z_{p}$
}

\author{
Ann VERDOODT
}

\begin{abstract}
Let $K$ be a non-archimedean valued field which contains $Q$, and suppose that $K$ is complete for the valuation $|\cdot|$, which extends the p-adic valuation. $V_{q}$ is the closure of the set $\left\{a q^{n} \mid n=\right.$ $0,1,2, \ldots\}$ where $a$ and $q$ are two units of $\boldsymbol{Z}_{p}, q$ not a root of unity. $C\left(V_{q} \rightarrow K\right)$ (resp. $C^{1}\left(V_{q} \rightarrow K\right)$ ) is the Banach space of continuous functions (resp. continuously differentiable functions) from $V_{q}$ to $K$. Our aim is to find orthonormal bases for $C\left(V_{q} \rightarrow K\right)$ and $C^{1}\left(V_{q} \rightarrow K\right)$.
\end{abstract}

\section{Introduction}

The main aim of this paper is to find orthonormal bases for the spaces $C\left(V_{q} \rightarrow K\right)$ of continuous and $C^{1}\left(V_{q} \rightarrow K\right)$ of continuously differentiable functions. Therefore we start by recalling some definitions and some previous results. Let $E$ be a non-archimedean Banach space over a non-archimedean valued field $L, E$ equipped with the norm \| - |.Let $f_{1}, f_{2}, \ldots$ be a finite or infinite sequence of elements of $E$. We say that this sequence is orthogonal if $\left\|\alpha_{1} f_{1}+\ldots+\alpha_{k} f_{k}\right\|=\max _{1 \leq i \leq k}\left\{\left\|\alpha_{i} f_{i}\right\|\right\}$ for all $k$ in $N$ (or for all $\mathrm{k}$ that do not exceed the length of the sequence) and for all $\alpha_{1}, \ldots, \alpha_{k}$ in $L$. An orthogonal sequence $f_{1}, f_{2}, \ldots$ is called orthonormal if $\left\|f_{i}\right\|=1$ for all $i$. A sequence $f_{1}, f_{2}, \ldots$ of elements of $E$ is an orthonormal base of $E$ if the sequence is orthonormal and also a base. If $M$ is a non-empty compact subset of $L$ whithout isolated points,

1991 Mathematics Subject Classification:46S10

Servicio Publicaciones Univ. Complutense. Madrid, 1996. 
then $C(M \rightarrow L)$ is the Banach space of continuous functions from $M$ to $L$ equipped with the supremum norm $\|\cdot\|_{\infty}$. Let $\mathrm{f}$ be a function from $M$ to $L$. The first difference quotient $\phi_{1} f$ of the function $f$ is the function of two variables given by $\phi_{1} f(x, y)=\frac{f(x)-f(y)}{x-y}$ defined on $M \times M \backslash \Delta$ where $\Delta=\{(x, x) \mid x \in M\}$. We say that $f$ is continuously differentiable at a point $b \in M\left(f\right.$ is $C^{1}$ at $\left.b\right)$ if $\lim _{(x, y) \rightarrow(b, b)} \phi_{1} f(x, y)$ exists. The function $f$ is called continuously differentiable ( $f$ is a $C^{1}$ function ) if $f$ is continuously differentiable at $b$ for all $b$ in $M$. If $f$ is a function from $M$ to $L$ then $f$ is continuously differentiable if and only if the function $\phi_{1} f$ can (uniquely) be extended to a continuous function on $M \times M$. The set of all $C_{1}$-functions from $M$ to $L$ is denoted by $C^{1}(M \rightarrow L)$, and $C^{1}(M \rightarrow L) \subset C(M \rightarrow L)$. For $f: M \rightarrow L$ we set $\|f\|_{1}=\sup \left\{\|f\|_{\infty},\left\|\phi_{1} f\right\|_{\infty}\right\}$. The function $\|\cdot\|_{1}$ is a norm on $C^{1}(M \rightarrow L)$ making it into an $L$-Banach algebra. Since $M$ is compact, $\|f\|_{1}<\infty$ if $f$ is an element of $C^{1}(M \rightarrow L)$ (these results concerning continuously differentiable functions can be found in [2] or [5], chapter 27).

Let $\boldsymbol{Z}_{p}$ be the ring of $\mathrm{p}$-adic integers, $Q_{p}$ the field of $\mathbf{p}$-adic numbers, and $K$ is a non-archimedean valued field, $K$ containing $Q_{p}$, and we suppose that $K$ is complete for the valuation $|\cdot|$, which extends the p-adic valuation. $\mathbb{N}$ denotes the set of natural numbers, and $\mathbb{N}_{0}$ is the set of natural numbers without zero. Let $a$ and $q$ be two units of $\boldsymbol{Z}_{p}, q$ not a root of unity. We define $V_{q}$ to be the closure of the set $\left\{a q^{n} \mid n=0,1,2, \ldots\right\}$. For a description of the set $V_{q}$ we refer to [7], section 2 or to [8], section 3. In section 3 our aim is to find orthonormal bases for the Banach space $C\left(V_{q} \rightarrow K\right)$. The results in section 3 can be seen as a sequel to the results in [9] and [8], sections 4,5 and 6. In section 4 we give necessary and sufficient conditions for a function $f$ in $C\left(V_{q} \rightarrow K\right)$ to be continuously differentiable, and we find an orthonormal base for the Banach space $C^{1}\left(V_{q} \rightarrow K\right)$.

Acknowledgement : I want to thank professor Van Hamme for the advice he gave me during the preparation of this paper.

\section{Preliminaries}

Let, us introduce the following :

$[n] !=[n][n-1] \ldots[1]$ and $[0] !=1$, where $[n]=\frac{q^{n}-1}{q-1}$ if $n \geq 1$. 


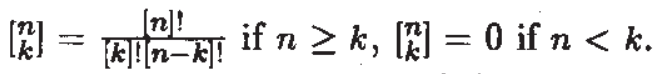

$\left\{\begin{array}{l}x \\ k\end{array}\right\}=\frac{(x-a)(x-a q) \ldots\left(x-a q^{k-1}\right)}{\left(a q^{k}-a\right)\left(a q^{k}-a q\right) \ldots\left(a q^{k}-a q^{k-1}\right)}$ if $k \geq 1,\left\{\begin{array}{l}x \\ 0\end{array}\right\}=1$.

The sequence $\left(\left\{\begin{array}{l}x \\ k\end{array}\right\}\right)$ forms an orthonormal base for $C\left(V_{q} \rightarrow K\right)$ ([8], corollary to lemma 8), analogous to Mahler's base for $C\left(\boldsymbol{Z}_{p} \rightarrow K\right)([4])$. We also have $\left[\begin{array}{l}n \\ k\end{array}\right]=\left\{\begin{array}{l}x \\ k\end{array}\right\}$ if $x=a q^{n}$. If $x$ is an element of $\phi_{p}$ with Henseldevelopment $x=\sum_{j=-\infty}^{+\infty} a_{j} p^{j}$, we then put $x_{n}=\sum_{j=-\infty}^{n-1} a_{j} p^{j}(n \in \mathbb{N})$. We write $m \triangleleft x$, if $\mathrm{m}$ is one of the numbers $x_{0}, x_{1}, \ldots$ and we say that " $\mathrm{m}$ is an initial part of $\mathrm{x}$ " or " $\mathrm{x}$ starts with $\mathrm{m}$ " (see [5], section 62). If $n$ belongs to $N_{0}, n=\sum_{j=0}^{s} a_{j} p^{j}$ where $a_{s} \neq 0$, then we put $n_{-}=\sum_{j=0}^{s-1} a_{j} p^{j}$. We remark that $n_{-} \triangleleft n$. Let us now define the sequence of functions $\left(e_{k}(x)\right)$ in the following way : write $k \in \mathbb{N}$ in the form $k=i+m j$, $0 \leq i<m(i, j \in \mathbb{N})$. Then $e_{k}$ is defined by

$e_{k}(x)=e_{i+m j}(x)=1$ if $x=a q^{i_{x}}\left(q^{m}\right)^{\alpha_{x}}$ where $i_{x}=i, j \triangleleft \alpha_{x}, e_{k}(x)=0$ otherwise.

The functions $\left(e_{k}(x)\right)$ form an orthonormal base for $C\left(V_{q} \rightarrow K\right)$ ([9]), analogous to van der Put's base for $C\left(Z_{p} \rightarrow K\right.$ ) (see [3] or [5], section 62).

We remark that $\left\{\begin{array}{l}a q^{j} \\ i\end{array}\right\}=e_{i}\left(a q^{j}\right)=0$ if $j<i$ and that $\left\{\begin{array}{l}a q^{i} \\ i\end{array}\right\}=e_{i}\left(a q^{i}\right)=1$. We shall use this frequently in the sequel.

We shall construct new orthonormal bases for $C\left(V_{q} \rightarrow K\right)$ using the bases $\left(\left\{\begin{array}{l}x \\ k\end{array}\right\}\right)$ and $\left(e_{k}(x)\right)$. Therefore we introduce the following: For each $n \in \mathbb{N}$, let $I_{n}$ be a subset of the set $\{0,1, \ldots, n\} \quad\left(I_{n}\right.$ can also be empty or can be equal to $\{0,1, \ldots, n\})$. Let $p(x)$ be a continuous function of the following type $p(x)=\sum_{i \in I_{n}} a_{i}\left\{\begin{array}{l}x \\ i\end{array}\right\}+\sum_{i \in\{0,1, \ldots, n\} \backslash I_{n}} a_{i} e_{i}(x)$ where each $a_{i} \in K$. For example, if $I_{n}=\{0,1, \ldots, n\}$, then $p(x)$ is a polynomial. If $I_{n}$ is the subset of $\{0,1, \ldots, n\}$ consisting of all the even numbers, and if $a_{i}=1$ for all $i$, then $p(x)=\sum_{i \in\{0,1, \ldots, n\}, i \text { even }}\left\{\begin{array}{l}\left.x^{x}\right\} \\ i \in\{0,1, \ldots, n\}, i \text { odd }\end{array} e_{i}(x)\right.$ and one can think of several other examples. For functions of this type we can prove the following lemmas

Lemma 1. Let $p(x)$ be a continuous function of the type $p(x)=\sum_{i \in I_{n}} a_{i}\left\{\begin{array}{l}x \\ i\end{array}\right\}+\sum_{i \in\{0,1, \ldots, n\} \backslash I_{n}} a_{i} e_{i}(x)\left(a_{i} \in K\right)$. Then the following are equivalent : 
1) $\left|p\left(a q^{n}\right)\right|=1$ and $\left|p\left(a q^{k}\right)\right|<1$ if $0 \leq k<n$.

2) $\left|a_{n}\right|=1$ and $\left|a_{k}\right|<1$ if $0 \leq k<n$.

\section{Proof.}

1) $\Rightarrow 2$ ) will be shown by induction. If $|p(a)|<1$ then $\left|a_{0}\right|<$ 1. Now suppose that $\left|a_{k}\right|<1$ if $0 \leq k<n-1$. Then $\left|\sum_{i \in I_{n} \cap\{0,1, \ldots k+1\}} a_{i}\left\{i^{a q^{k+1}}\right\}+\sum_{i \in\{0,1, \ldots, k+1\} \backslash I_{n}} a_{i} e_{i}\left(a q^{k+1}\right)\right|=\left|p\left(a q^{k+1}\right)\right|<$ 1 and by the induction hypothesis it follows that $\left|a_{k+1}\right|<1$ and we can conclude $\left|a_{i}\right|<1$ for all $0 \leq i<n$. Since $\mid \sum_{i \in I_{n}} a_{i}\left\{\begin{array}{l}a q^{n} \\ n^{n}\end{array}+\sum_{i \in\{0,1, \ldots, n\} \backslash I_{n}} a_{i} e_{i}\left(a q^{n}\right)|=| p\left(a q^{n}\right) \mid=1\right.$ we have $\left|a_{n}\right|=1$. 2) $\Rightarrow 1$ ) is obvious.

Lemma 2. Let $p(x)$ be a continuous function of the type $p(x)=\sum_{i \in I_{n}} a_{i}\left\{\begin{array}{l}x \\ i\end{array}\right\}+\sum_{i \in\{0,1, \ldots, n\} \backslash I_{n}} a_{i} e_{i}(x)\left(a_{i} \in K\right)$. Then the following are equivalent :

1) $\|p\|_{\infty} \leq 1$.

2) $\left|a_{k}\right| \leq 1$ for all $k$ with $0 \leq k \leq n$.

\section{Proof.}

1) $\Rightarrow 2$ ) can be shown analogous as 1) $\Rightarrow 2$ ) of the previous lemma. 2) $\Rightarrow 1$ ) is obvious.

Let $m$ be the smallest integer such that $q^{m} \equiv 1(\bmod p)(1 \leq m \leq p-1)$. There exists a $k_{0}$ such that $q^{m} \equiv 1\left(\bmod p^{k_{0}}\right), q^{m} \not \equiv 1\left(\bmod p^{k_{0}+1}\right)$. If $\left(p, k_{0}\right)=(2,1)$, i.e. $q \equiv 3(\bmod 4)$, then there exists a natural number $N$ such that $q=1+2+2^{2} \varepsilon, \varepsilon=\varepsilon_{0}+\varepsilon_{1} 2+\varepsilon_{2} 2^{2}+\ldots, \varepsilon_{0}=\varepsilon_{1}=\ldots=$ $\varepsilon_{N-1}=1, \varepsilon_{N}=0$. Then we have

\section{Lemma 3.}

1) Let $q^{m} \equiv 1\left(\bmod p^{k_{0}}\right), q^{m} \not \equiv 1\left(\bmod p^{k_{0}+1}\right)$ with $\left(p, k_{0}\right) \neq(2,1)$. If $x, y \in V_{q},|x-y| \leq p^{-\left(k_{o}+t\right)}$ then $e_{n}(x)=e_{n}(y)$ if $0 \leq n<m p^{t}$. 2) Let $q \equiv 3(\bmod 4), q=1+2+2^{2} \varepsilon, \varepsilon=\varepsilon_{0}+\varepsilon_{1} 2+\varepsilon_{2} 2^{2}+\ldots$, $\varepsilon_{0}=\varepsilon_{1}=\ldots=\varepsilon_{N-1}=1, \varepsilon_{N}=0$. If $x, y \in V_{q},|x-y| \leq p^{-(N+2+t)}$ then $e_{n}(x)=e_{n}(y)$ if $0 \leq n<2^{t}(t \geq 1)$.

Pro6f. This follows immediately from [8], lemmas 2 and 3. 
Lemma 4. Suppose $p(x)$ is a continuous function with $\|p\|_{\infty} \leq 1$ of the following type $: p(x)=\sum_{i \in I_{n}} a_{i}\left\{\begin{array}{l}x \\ i\end{array}\right\}+\sum_{i \in\{0,1, \ldots, n\} \backslash I_{n}} a_{i} e_{i}(x)\left(a_{i} \in K\right)$.

1) Let $q^{m} \equiv 1\left(\bmod p^{k_{0}}\right), q^{m} \not \equiv 1\left(\bmod p^{k_{0}+1}\right)$ with $\left(p, k_{0}\right) \neq(2,1)$. If $x, y \in V_{q},|x-y| \leq p^{-\left(k_{0}+t\right)}$ then if $j \in \mathbb{N}, 0 \leq n<m p^{t}: \mid p(x)^{j}-$ $p(y)^{j} \mid \leq 1 / p$ and $\left|x^{j}-y^{j}\right| \leq 1 / p$.

2) Let $q \equiv 3(\bmod 4), q=1+2+2^{2} \varepsilon, \varepsilon=\varepsilon_{0}+\varepsilon_{1} 2+\varepsilon_{2} 2^{2}+\ldots$, $\varepsilon_{0}=\varepsilon_{1}=\ldots=\varepsilon_{N-1}=1, \varepsilon_{N}=0$. If $x, y \in V_{q},|x-y| \leq p^{-(N+2+t)}$ then if $j \in \mathbb{N}, 0 \leq n<2^{t}(t \geq 1):\left|p(x)^{j}-p(y)^{j}\right| \leq 1 / 2$ and $\left|x^{j}-y^{j}\right|^{j} \leq 1 / 2$.

Proof. It is clear that $\left|a_{s}\right| \leq 1$ if $0 \leq s \leq n$ (lemma 2). Suppose that $x, y$ and $n$ are as in 1) (resp. 2)). Then $|p(x)-p(y)| \leq \max _{s \in I_{n}}\left\{\left|a_{s}\right| \mid\left\{\begin{array}{l}x \\ s\end{array}\right\}-\right.$ $\left\{\begin{array}{l}y \\ s\end{array} \mid\right\} \leq 1 / p$ (resp. $\leq 1 / 2$ ) by lemma 3 and $[8]$, lemmas 11 and 12 . If $j>1$ then $\left|p(x)^{j}-p(y)^{j}\right|=|p(x)-p(y)|\left|\sum_{s=0}^{j-1} p(x)^{s} p(y)^{j-1-s}\right| \leq 1 / p$ (resp. $\leq 1 / 2$ ). So the lemma holds for $j \in \mathbb{N}$. (the case $j=0$ is trivial). Further, if $j>1$ then $\left|x^{j}-y^{j}\right| \leq|x-y|\left|\sum_{s=0}^{j-1} x^{s} y^{j-1-s}\right| \leq 1 / p$ (resp. $\leq 1 / 2$ ) so $\left|x^{j}-y^{j}\right| \leq 1 / p$ (resp. $\left.\leq 1 / 2\right)$ for all $j \in \mathbb{N}$.

Let for each $n \in \mathbb{N} J_{n}$ be a subset of the set $\{0,1, \ldots, n\}$. Then we can prove

Lemma 5. Let $p(x)$ and $q(x)$ be continuous functions with $\|\dot{p}\|_{\infty} \leq 1$ and $\|q\|_{\infty} \leq 1$ of the form $p(x)=\sum_{i \in I_{n}}^{\infty} a_{i}\left\{\begin{array}{l}x \\ i\end{array}+\sum_{i \in\{0,1, \ldots, n\} \backslash I_{n}} a_{i} e_{i}(x),\left(a_{i} \in K\right)\right.$ $q(x)=\sum_{i \in J_{n}} b_{i}\left\{\begin{array}{l}x \\ i\end{array}\right\}+\sum_{i \in\{0,1, \ldots, n\} \backslash J_{n}} b_{i} e_{i}(x),\left(b_{i} \in K\right)$.

1) Let $q^{m} \equiv 1\left(\bmod p^{k_{0}}\right), q^{m} \not \equiv 1\left(\bmod p^{k_{0}+1}\right)$ with $\left(p, k_{0}\right) \neq(2,1)$. If $x, y \in V_{q},|x-y| \leq p^{-\left(k_{0}+t\right)}$ then if $i, j \in \mathbb{N}, 0 \leq n<m p^{t}: \mid q(x)^{i} p(x)^{j}-$ $q(y)^{i} p(y)^{j} \mid \leq 1 / p$ and $\left|x^{i} p(x)^{j}-y^{i} p(x)^{j}\right| \leq 1 / p$.

2) Let $q \equiv 3(\bmod 4), q=1+2+2^{2} \varepsilon, \varepsilon=\varepsilon_{0}+\varepsilon_{1} 2+\varepsilon_{2} 2^{2}+\ldots$, $\varepsilon_{0}=\varepsilon_{1}=\ldots=\varepsilon_{N-1}=1, \varepsilon_{N}=0$. If $x, y \in V_{q},|x-y| \leq p^{-(N+2+t)}$ then if $i, j \in \mathbb{N}, 0 \leq n<2^{t}(t \geq 1):\left|q(x)^{i} p(x)^{j}-q(y)^{i} p(y)^{j}\right| \leq 1 / 2$ and $\left|x^{i} p(x)^{j}-y^{i} p(x)^{j}\right| \leq 1 / 2$.

Proof. Let $x, y, n, i$ and $j$ be as in 1) (resp. 2)) then 
$\left|q(x)^{i} p(x)^{j}-q(y)^{i} p(y)^{j}\right| \leq \max \left\{\left|q(x)^{i} p(x)^{j}-q(x)^{i} p(y)^{j}\right|, \mid q(x)^{i} p(y)^{j}-\right.$ $\left.q(y)^{i} p(y)^{j} \mid\right\}$

$\leq \max \left\{\left|q(x)^{i}\right|\left|p(x)^{j}-p(y)^{j}\right|,\left|p(y)^{j}\right|\left|q(x)^{i}-q(y)^{i}\right|\right\}$

$\leq 1 / p$ (resp. $\leq 1 / 2$ ) by lemma 5 and analogous

$\left|x^{i} p(x)^{j}-y^{i} p(y)^{j}\right| \leq \max \left\{\left|x^{i} p(x)^{j}-x^{i} p(y)^{j}\right|,\left|x^{i} p(y)^{j}-y^{i} p(y)^{j}\right|\right\}$

$\leq \max \left\{\left|x^{i}\right|\left|p(x)^{j}-p(y)^{j}\right|,\left|p(y)^{j}\right|\left|x^{i}-y^{i}\right|\right\}$

$\leq 1 / p($ resp. $\leq 1 / 2)$ by lemma 5

We shall need lemmas 6 and 7 for the construction of an orthonormal base for $C^{1}\left(V_{q} \rightarrow K\right)$ :

Lemma 6.

$\left[\begin{array}{l}i+j \\ n\end{array}\right]=\sum_{s=0}^{n}\left[\begin{array}{l}j \\ n-s\end{array}\right]\left[\begin{array}{l}i \\ s\end{array}\right] q^{-(n-s)(-i+s)}$

Proof. This follows immediately from [8], lemma 10 by putting first $s=n-k$ and then interchanging $i$ and $j$.

Definition. We define the sequence $\left(\rho_{n}\right)$ as follows :

$\rho_{n}=\left(q^{m}\right)^{i-i-}-1$ if $n=i m+j, 0 \leq j<m$ and $i>0, \rho_{n}=1$ if $n<m$.

Lemma 7.

$\left|\rho_{n}\right|=\min _{1 \leq s \leq n}\left\{\left|q^{s}-1\right|\right\} .\left(n \in \mathbb{N}_{0}\right)$.

Proof. This follows immediately from [8], lemmas 2 and 3.

\section{Orthonormal bases for $C\left(V_{q} \rightarrow K\right)$}

Using the lemmas $1-5$ in section 2, we can make orthonormal bases for $C\left(V_{q} \rightarrow K\right)$ with the aid of the following theorem :

Theorem 1. Let $\left(p_{n}(x)\right)$ and $\left(q_{n}(x)\right)$ be sequences of continuous functions of the following form:

$$
\begin{aligned}
& \text { for each } n p_{n}(x) \text { is of the form } p_{n}(x)=\sum_{i \in I_{n}} a_{n, 1}\left\{\begin{array}{l}
x \\
i
\end{array}\right\}+ \\
& \sum_{i \in\{0,1, \ldots, n\} \backslash I_{n}} a_{n, i} e_{i}(x) \text { with }\left|a_{n, n}\right|=1 \text { and with }\left|a_{n, i}\right|<1 \\
& \text { if } 0 \quad i_{i}<n \quad\left(a_{n, i} \in \Phi_{p}\right), \text { and for each } n \text { we have } \\
& q_{n}(x)=\sum_{i \in J_{n}} b_{n, i}\left\{\begin{array}{l}
x \\
i
\end{array}\right\}+\sum_{i \in\{0,1, \ldots, n\} \backslash J_{n}} b_{n, i} e_{i}(x) \text { with }\left|q_{n}\left(a q^{n}\right)\right|=1 \text { and }
\end{aligned}
$$


$\left|b_{n, i}\right| \leq 1$ if $0 \leq i \leq n\left(b_{n, i} \in \mathbb{Q}_{p}\right)$. If $\left(j_{n}\right)$ is a sequence in $\mathbb{N}$ and if $\left(k_{n}\right)$ is a sequence in $N_{0}$, then the sequences $\left(q_{n}(x)^{j_{n}} p_{n}(x)^{k_{n}}\right)$ and $\left(x^{j_{n}} p_{n}(x)^{k_{n}}\right)$ form orthonormal bases for $C\left(V_{q} \rightarrow K\right)$.

Proof. This proof is analogous to the proof of $[8]$, theorem 5 . We remark that for all $n$ we have $\left\|p_{n}\right\|_{\infty} \leq 1$ and $\left\|q_{n}\right\|_{\infty} \leq 1$ (lemma 2), and that $p_{n}(x)$ and $q_{n}(x)$ are elements of $C\left(V_{q} \rightarrow Q_{p}\right)$. By [1], 3.4.1 or [6], p. $123-133$ it suffices to prove that $\left(q_{n}(x)^{j_{n}} p_{n}(x)^{k_{n}}\right)$ and $\left(x^{j_{n}} p_{n}(x)^{k_{n}}\right)$ form orthonormal bases for $C\left(V_{q} \rightarrow Q_{q}\right)$ and by [1] proposition 3.1 .5 p. 82 it suffices to prove that $\overline{\left(q_{n}(x)^{j_{n}} p_{n}(x)^{k_{n}}\right.}$ and $\left.\overline{\left(x^{j_{n}} p_{n}(x)^{k_{n}}\right.}\right)$ form vectorial bases for $C\left(V_{q} \rightarrow \boldsymbol{F}_{p}\right)$ (where $\overline{f(x)}$ stands for the canonical projection on $C\left(V_{q} \rightarrow \boldsymbol{F}_{p}\right)$, if $\mathrm{f}$ is in $C\left(V_{q} \rightarrow Q_{p}\right)$ with $\left.\|f\|_{\infty} \leq 1\right)$. We distinguish two cases.

1) Let $q^{m} \equiv 1\left(\bmod p^{k_{0}}\right), q^{m} \not \equiv 1\left(\bmod p^{k_{0}+1}\right)$ with $\left(p, k_{0}\right) \neq(2,1)$, define $C_{t}$ the space of the functions from $V_{q}$ to $F_{p}$ constant on balls of the type $\left\{x \in \mathcal{Z}_{p}:|x-\alpha| \leq p^{-\left(k_{0}+t\right)}\right\}, \alpha \in V_{q}$. Since $C\left(V_{q} \rightarrow F_{p}\right)=\cup_{t<0} C_{t}([8]$, lemma 4 and its proof) it suffices to prove that $\left(\overline{q_{n}(x)^{j_{n}} p_{n}(x)^{k_{n}}} \mid n<m p^{t}\right)$ and $\left(\overline{x^{j_{n}} p_{n}(x)^{k_{n}}} \mid n<m p^{t}\right)$ form bases for $C_{t}$. By the proof of [8], lemma 4 , we can write $V_{q}$ as the union of $m p^{t}$ disjoint balls with radius $p^{-\left(k_{0}+t\right)}$ and with centers $a q^{r}\left(q^{m}\right)^{n}, 0 \leq r \leq m-1,0 \leq n<p^{t}$. Let $\chi_{i}$ be the characteristic function of the ball with center $a q^{i}$. Using lemma 5 , we have

$$
\begin{aligned}
\overline{q_{n}(x)^{j_{n}} p_{n}(x)^{k_{n}}} & =\sum_{i=0}^{m p^{t}-1} \chi_{i}(x) \overline{q_{n}\left(a q^{i}\right)^{j_{n}} p_{n}\left(a q^{i}\right)^{k_{n}}} \\
& =\sum_{i=n}^{m p^{t}-1} \chi_{i}(x) \overline{q_{n}\left(a q^{i}\right)^{j_{n}} p_{n}\left(a q^{i}\right)^{k_{n}}}
\end{aligned}
$$

since $\left|q_{n}\left(a q^{i}\right)^{j_{n}} p_{n}\left(a q^{i}\right)^{k_{n}}\right|<1$ if $i<n$ (lemma 1) and hence the transition matrix from $\left(\chi_{n} \mid n<m p^{t}\right)$ to $\left(\overline{q_{n}(x)^{j_{n}} p_{n}(x)^{k_{n}}} \mid n<m p^{t}\right)$ is triangular since $\left|q_{n}\left(a q^{n}\right)^{j_{n}} p_{n}\left(a q^{n}\right)^{k_{n}}\right|=1$ (lemma 1), so $\left(\overline{q_{n}(x)^{j_{n}} p_{n}(x)^{k_{n}}} \mid n<m p^{t}\right.$ ) forms a base for $C_{t}$. The proof for $\left(\overline{x^{j_{n}} p_{n}(x)^{k_{n}}}\right)$ is analogous.

2) Let $q^{m} \equiv 3(\bmod 4), q=1+2+2^{2} \varepsilon, \varepsilon=\varepsilon_{0}+\varepsilon_{1} 2+\varepsilon_{2} 2^{2}+\ldots, \varepsilon_{0}=$ $\varepsilon_{1}=\ldots=\varepsilon_{N-1}=1, \varepsilon_{N}=0$, define $C_{t}$ te space of the functions from $V_{q}$ to $\boldsymbol{F}_{2}$ constant on balls of the type $\left\{x \in \mathbb{Z}_{2}:|x-\alpha| \leq 2^{-(N+2+t)}\right\}$, $\alpha \in V_{q}$. Since $C\left(V_{q} \rightarrow F_{2}\right)=\cup_{t \geq 1} C_{t}([8]$, lemma 5 and its proof) it 
suffices to prove that $\left(\overline{q_{n}(x)^{j_{n}} p_{n}(x)^{k_{n}}} \mid n<2^{t}\right)$ and $\left.\overline{\left(x^{j_{n}} p_{n}(x)^{k_{n}}\right.} \mid n<2^{t}\right)$ form bases for $C_{t}$. By the proof of [8], lemma 5 , we can write $V_{q}$ as the union of $2^{t}$ disjoint balls with radius $2^{-(N+2+t)}$ and with centers $a q^{n}$, $0 \leq n<2^{t}$. From now on the proof is analogous to the proof of 1 ).

\section{Some examples.}

1) If $\left(p_{n}(x)\right)$ is a sequence of polynomials with coefficients in $Q_{p}$ such that for all $n$ we have that the degree of $p_{n}$ is $n,\left|p_{n}\left(a q^{n}\right)\right|=1$ and $\left|p_{n}\left(a q^{i}\right)\right|<1$ if $0 \leq i<n$, and if $\left(k_{n}\right)$ is a sequence in $\mathbb{N}_{0}$, then $\left(p_{n}(x)^{k_{n}}\right)$ forms an orthonormal base for $C\left(V_{q} \rightarrow K\right)$. This follows immediately from lemma 1 and theorem 1 , by putting $j_{n}=0$ and $I_{n}=\{0,1, \ldots n\}$ and this for all $n$. The case $k_{n}=1$ for all $n$ can also be found in [8], theorem 4.

2) If $\left(k_{n}\right)$ is a sequence in $\mathbb{N}_{0}$, then $\left(\left\{\begin{array}{l}x \\ n\end{array}\right\}^{k_{n}}\right)$ forms an orthonormal base for $C\left(V_{q} \rightarrow K\right)$. Put therefore $p_{n}(x)=\left\{\begin{array}{l}x \\ n\end{array}\right\}$ in 1). If $f$ is an element of $C\left(V_{q} \rightarrow K\right)$, and if $s$ is a natural number different from zero, there exists a uniformly convergent expansion $f(x)=\sum_{n=0}^{\infty} \beta_{n}^{(s)}\left\{\begin{array}{l}x \\ n\end{array}\right\}^{s}$ and we are able to give an expression for the coeffiecients $\beta_{n}^{(s)}$. This can be found in [8], proposition 1 .

3) If $\left(p_{n}(x)\right)$ is a sequence in $C\left(V_{q} \rightarrow \Phi_{p}\right)$ such that for all $n$ we have $p_{n}(x)=\sum_{i=0}^{n} a_{n, i} e_{i}(x)$ with $\left|p_{n}\left(a q^{n}\right)\right|=1$ and $\left|p_{n}\left(a q^{i}\right)\right|<1$ if $0 \leq i<n$, and if $\left(k_{n}\right)$ is a sequence in $N_{0}$, then $\left(p_{n}(x)^{k_{n}}\right)$ forms an orthonormal base for $C\left(V_{q} \rightarrow K\right)$. This follows immediately from lemma 1 and theorem 1 , by putting $j_{n}=0$ and by putting $I_{n}$ equal to the empty set. The case $k_{n}=1$ for all $n$ can also be found in [9], theorem 2 .

Remark. We can make an analogous result for the space $C\left(\boldsymbol{Z}_{p} \rightarrow K\right)$ : if we replace the polynomials $\left(\left\{\begin{array}{l}x \\ i\end{array}\right\}\right)$ by $\left(\left(\begin{array}{l}x \\ i\end{array}\right)\right)$ (Mahler's base) and the functions $\left(e_{i}(x)\right)$ by van der Put's base, then we can prove the following (we shall denote van der Put's base by $\left(g_{i}(x)\right)$ :

Let $\left(p_{n}(x)\right)$ and $\left(q_{n}(x)\right)$ be sequences of continuous functions on $\boldsymbol{Z}_{p}$ of the following form: for each $n p_{n}(x)$ is of the form $p_{n}(x)=\sum_{i \in I_{n}} a_{n, i}\left(\begin{array}{l}x \\ i\end{array}\right)+\sum_{i \in\{0,1, \ldots, n\} \backslash I_{n}} a_{n, i} g_{i}(x)$ with $\left|a_{n, n}\right|=1$ and with $\left|a_{n, i}\right|<1$ if $0 \leq i<n\left(a_{n, i} \in Q_{p}\right)$, and for each $n$ we have 
$q_{n}(x)=\sum_{i \in J_{n}} b_{n, i}\left(\begin{array}{l}x \\ i\end{array}\right)+\sum_{i \in\{0,1, \ldots, n\} \backslash J_{n}} b_{n, i} g_{i}(x)$ with $\left|q_{n}(n)\right|=1$ and $\left|b_{n, i}\right| \leq$ 1 if $0 \leq i \leq n\left(b_{n, i} \in \Phi_{p}\right)$. If $\left(j_{n}\right)$ is a sequence in $N$ and if $\left(k_{n}\right)$ is a sequence in $N_{0}$, then the sequence $\left(q_{n}(x)^{j_{n}} p_{n}(x)^{k_{n}}\right)$ forms an orthonormal base for $C\left(\mathcal{Z}_{p} \rightarrow K\right)$.

\section{Continuously differentiable functions on $V_{q}$}

In this section we give necessary and sufficient conditions for a continuous function defined on $V_{q}$ to be continuously differentiable, and we find an orthonormal base for the space $C^{1}\left(V_{q} \rightarrow K\right)$. The result we'll find is analogous to the result for continuously differentiable functions on $\boldsymbol{Z}_{p}$ ([5], theorem 53.5) where we replace Mahler's base by the base $\left(\left\{\begin{array}{l}x \\ n\end{array}\right\}\right)$. We remark that there is a one-to-one correspondence between $(u, v) \in V_{q} \times V_{q}$ and $\left(\frac{q y x}{a}, x\right)$ with $(x, y) \in V_{q} \times V_{q}$ (see [7], section 2). We shall use this several times in this section. Let $\rho_{n}$ be as defined in section 2, then we can prove the following :

Proposition 1. Let $f$ be an element of $C\left(V_{q} \rightarrow K\right)$ with uniformly convergent expansion $f(x)=\sum_{n=0}^{\infty} a_{n}\left\{\begin{array}{l}x \\ n\end{array}\right\}$. If $\operatorname{tim}_{n \rightarrow \infty}\left|a_{n}\left(\rho_{n}\right)^{-1}\right|=0$, then $f$ is an element of $C^{1}\left(V_{q} \rightarrow K\right)$.

Proof. Let $f$ be in $C\left(V_{q} \rightarrow K\right)$ with uniformly convergent expansion $f(x)=\sum_{n=0}^{\infty} a_{n}\left\{\begin{array}{l}x \\ n\end{array}\right\}$. Analogous to [5], theorems 53.4 and 53.5 , we want to find an expression for $\phi_{1} f(u, v)$ for special values for $u$ and $v$. Therefore, let $x, y$ be in $\left\{a q^{n} \mid n=0,1,2, \ldots\right\}$, $x=a q^{i}, y=a q^{j}$ and suppose $y \neq a$ (i.e. $j \neq 0$ ). Then $\phi_{1} f\left(\frac{y x}{a}, x\right)=\phi_{1} f\left(x, \frac{y x}{a}\right)=\frac{f\left(\frac{y x}{a}\right)-f(x)}{\frac{p_{x}}{a}-x}=\sum_{n=1}^{\infty} \frac{a_{n}}{a q^{i}\left(q^{j}-1\right)}\left(\left[\begin{array}{l}i+j_{1} \\ n\end{array}-\left[\begin{array}{l}i \\ n\end{array}\right]\right)\right.$ $\left.\left.=\sum_{n=1}^{\infty} \frac{\underline{a_{n}}}{a q^{i}\left(q^{j}-1\right)}\left(\sum_{s=0}^{n} l_{n-s}^{j}\right] l_{s}^{i}\right] q^{-(n-s)(-i+s)}-\left[\begin{array}{l}i \\ n\end{array}\right]\right)$ (by lemma 6$)$ $=\sum_{n=1}^{\infty} \frac{a_{n}}{a q^{i}\left(q^{j}-1\right)} \sum_{s=0}^{n-1}\left[_{n-s}^{j}\right]\left[\begin{array}{l}i \\ s\end{array}\right] q^{-(n-s)(-i+s)}$ since $\frac{1}{q^{j}-1}\left[\begin{array}{l}j \\ n-s\end{array}\right]=\frac{1}{q^{n-s}-1}\left[\begin{array}{l}j-1 \\ n-s-1\end{array}\right]$, we find, by putting $n=s+k+1$, that 
$\phi_{1} f\left(\frac{\dot{y} x}{a}, x\right)=\sum_{k=0}^{\infty} \sum_{s=0}^{\infty} \frac{a_{k+s+1} q^{-s(k+1)}}{a^{k+1}\left(q^{k+1}-1\right)} x^{k}\left\{\begin{array}{l}x \\ s\end{array}\right\}\left\{\begin{array}{l}y / q \\ k\end{array}\right\}$

and replacing $y$ by $y q$ this gives us, for all $x, y$ in $\left\{a q^{n} \mid n=0,1,2, \ldots\right\}$

$\phi_{1} f\left(\frac{q y x}{a}, x\right)=\sum_{k=0}^{\infty} \sum_{s=0}^{\infty} \frac{a_{k+s+1} q^{-s(k+1)}}{a^{k+1}\left(q^{k+1}-1\right)} x^{k}\left\{\begin{array}{l}x \\ s\end{array}\right\}\left\{\begin{array}{l}y \\ k\end{array}\right\}\left(^{*}\right)$

Now $\sup _{k+s+1=n}\left|\frac{a_{k+s+1}}{q^{k+1}-1}\right|=\left|a_{n}\right| \max _{1 \leq k \leq n}\left|\frac{1}{q^{k}-1}\right|=\left|a_{n}\left(\rho_{n}\right)^{-1}\right|$ (lemma 7), so if $\lim _{n \rightarrow \infty}\left|a_{n}\left(r_{n}\right)^{-1}\right|=0$, then $\lim _{k+s \rightarrow \infty}\left|\frac{a_{k+s+1}}{q^{k+1}-1}\right|=0$ and it is clear that $\left(^{*}\right)$ can be extended to a continuous function ([5], exercise 23.B). So we conclude : if $\lim _{n \rightarrow \infty}\left|a_{n}\left(r_{n}\right)^{-1}\right|=0$, then $f \in C^{1}\left(V_{q} \rightarrow K\right)$. This finishes the proof.

Remark. It is easy to prove that the functions $\left(x^{k}\left\{\begin{array}{c}x \\ s\end{array}\right\}\left\{\begin{array}{c}y \\ k\end{array}\right\}\right)$ are orthonormal in $C\left(V_{q} \times V_{q} \rightarrow K\right)$.

Let $A$ be the subset of $C\left(V_{q} \rightarrow K\right)$ defined as follows : if $\mathrm{f}$ is an element of $C\left(V_{q} \rightarrow K\right)$ with uniformly convergent expansion $f(x)=\sum_{n=0}^{\infty} a_{n}\left\{\begin{array}{l}x \\ n\end{array}\right\}$, then $f$ is an element of $A$ if and only if $\lim _{n \rightarrow \infty}\left|a_{n}\left(\rho_{n}\right)^{-1}\right|=0$.

Proposition 2. The set A satisfies the following properties:

1) $A$ is a subset of $C^{1}\left(V_{q} \rightarrow K\right)$ containing the polynomials 2) $A$ is closed for $\|\cdot\|_{1}$ 3) $A$ is a subalgebra of $C^{1}\left(V_{q} \rightarrow K\right)$

\section{Proof.}

1) From proposition 1 it follows that $A$ is a subset of $C^{1}\left(V_{q} \rightarrow K\right)$. It is clear that $A$ contains the polynomials.

2) Suppose $f=\lim _{n \rightarrow \infty} f_{n}$ for the norm $\|\cdot\|_{1}$ where $f_{n} \in A$ for all $n$. Then $f$. is clearly continuous. So there exists the following uniformly convergent expansions : $f(x)=\sum_{k=0}^{\infty} a_{k}\left\{\begin{array}{l}x \\ k\end{array}\right\}, f_{n}(x)=\sum_{k=0}^{\infty} a_{n, k}\left\{\begin{array}{l}x \\ k\end{array}\right\}$, with $\lim _{k \rightarrow \infty}\left|a_{k}\right|=0, \lim _{k \rightarrow \infty}\left|a_{n, k}\right|=0$ for all $n, \lim _{k \rightarrow \infty}\left|a_{n, k}\left(\rho_{k}\right)^{-1}\right|=$ 0 for all $n$. Suppose that $\lim _{k \rightarrow \infty}\left|a_{k}\left(\rho_{k}\right)^{-1}\right| \neq 0$. This will lead to a contradiction. Since $\lim _{k \rightarrow \infty}\left|a_{k}\left(\rho_{k}\right)^{-1}\right| \neq 0$ there exists an $\epsilon>0$ such that for all $\eta \in \mathbb{N}$, there exists an $n>\eta$ such that $\left|a_{n}\left(\rho_{n}\right)^{-1}\right|>\epsilon$. Let $I$ be the set defined as follows : $I=\left\{k \in N_{0}:\left|a_{k}\left(\rho_{k}\right)^{-1}\right|>\epsilon\right\}$. Then $I$ is infinite. Let $\epsilon$ be as above. Then there exists a $J \in \mathbb{N}$, such that for all $n \geq$ $J$ we have $\left\|f-f_{n}\right\|_{1}<\epsilon$. In particular, $\sup _{x \neq y}\left\{\left|\frac{\left(f-f_{J}\right)(x)-\left(f-f_{J}\right)(y)}{x-y}\right|\right\}<$ $\epsilon$, and from the calculations in proposition 1 it follows that 
$\left|\phi_{1}\left(f-f_{J}\right)\left(\frac{q y x}{a}, x\right)\right|=\left|\sum_{k=0}^{\infty} \sum_{s=0}^{\infty} \frac{\left(a_{k+s+1}-a_{J, k+s+1}\right) q^{-s(k+1)}}{a^{k+1}\left(q^{k+1}-1\right)} x^{k}\left\{\begin{array}{l}x \\ s\end{array}\right\}\left\{\begin{array}{l}y \\ k\end{array}\right\}\right| \leq$ $\epsilon$ for all $x, y$ in $\left\{a q^{n} \mid n=0,1,2, \ldots\right\}$. From this it is easy to see that $\left|\frac{a_{k+s+1}-a_{J, k+s+1}}{q^{k+1}-1}\right| \leq \epsilon$ for all $k$ and $s$, so $\sup _{k, s}\left\{\frac{a_{k+s+1}-a_{J, k+s+1}}{q^{k+1}-1} \mid\right\} \leq \epsilon$ and thus $\sup _{n}\left\{\left|\left(a_{n}-a_{J, n}\right)\left(\rho_{n}\right)^{-1}\right|\right\} \leq \epsilon$. Then, if $n \in I$ we have $\left|a_{J, n}\left(\rho_{n}\right)^{-1}\right|=$ $\left|\left(a_{J, n}-a_{n}\right)\left(\rho_{n}\right)^{-1}+a_{n}\left(\rho_{n}\right)^{-1}\right|>\epsilon$, and from this it follows that $\lim _{k \rightarrow \infty}$ $\left|a_{J, k}\left(\rho_{k}\right)^{-1}\right| \neq 0$ since $I$ is infinite. This is impossible and we conclude that $A$ is closed.

3) If $f, g \in A, k, j \in K$, then we immediately have that $k f+j g \in A$, and if $r$ and $u$ are polynomials ( $\epsilon A$ ) then $r u$ is a polynomial and also an element of $A$. From the Weierstrass-theorem for $C^{1}$-functions ([2], theorem 1.4) it follows that for each $f, g \in A$ we have $f g \in A$ since $A$ is closed.

Theorem 2. Let $f$ be an element of $C\left(V_{q} \rightarrow K\right)$ with uniformly convergent expansion $f(x)=\sum_{n=0}^{\infty} a_{n}\left\{\begin{array}{l}x \\ n\end{array}\right\}$. Then $f$ is an element of $C^{1}\left(V_{q} \rightarrow K\right)$ if and only if $\lim _{n \rightarrow \infty}\left|a_{n}\left(\rho_{n}\right)^{-1}\right|=0$.

If $f$ is an element of $C^{1}\left(V_{q} \rightarrow K\right)$ then $\|f\|_{1}=\max _{n \geq 0}\left\{\left|a_{n}\left(\rho_{n}\right)^{-1}\right|\right\}$ and the functions $\left(\rho_{n}\left\{\begin{array}{l}x \\ n\end{array}\right\}\right)$ form an orthonormal base for $C^{1}\left(V_{q} \rightarrow K\right)$.

Proof. From proposition 2 and the Weierstrass-Stone theorem for $C^{1}$ functions ([2], theorem 2.10) it follows that $A=C^{1}\left(V_{q} \rightarrow K\right)$. So $f$ is an element of $A=C^{1}\left(V_{q} \rightarrow K\right)$ if and only if $\lim _{n \rightarrow \infty}\left|a_{n}\left(\rho_{n}\right)^{-1}\right|=0$. Let us first remark the following : since $\lim _{n \rightarrow \infty}\left|a_{n}\left(\rho_{n}\right)^{-1}\right|=0$, we have $\sup _{n \geq 1}\left\{\left|a_{n}\left(\rho_{n}\right)^{-1}\right|\right\}=$ $\max _{n \geq 1}\left\{\left|a_{n}\left(\rho_{n}\right)^{-1}\right|\right\}$ and since $\sup _{k, s \geq 0}\left\{\left|\frac{a_{k+s+1}}{q^{k+1}-1}\right|\right\}=\sup _{n \geq 1}\left\{\left|a_{n}\left(\rho_{n}\right)^{-1}\right|\right\}$ with $k+s+1=n$, we have

$\max _{k, s \geq 0}\left\{\left|\frac{a_{k+a+1}}{q^{k+1}-1}\right|\right\}=\sup _{k, s \geq 0}\left\{\left|\frac{a_{k+s+1}}{q^{k+1}-1}\right|\right\}=\max _{n \geq 1}\left\{\left|a_{n}\left(\rho_{n}\right)^{-1}\right|\right\}$. From $\left(^{*}\right)$ it follows that for all $x, y$ in $\left\{a q^{n} \mid n=0,1,2, \ldots\right\}$

$\phi_{1} f\left(\frac{q y x}{a}, x\right)=\sum_{k=0}^{\infty} \sum_{s=0}^{\infty} \frac{a_{k+s+1} q^{-s(k+1)}}{a^{k+1}\left(q^{k+1}-1\right)} x^{k}\left\{\begin{array}{l}x \\ s\end{array}\right\}\left\{\begin{array}{l}y \\ k\end{array}\right\}$ and by continuity it then follows that for all $x, y$ in $V_{q}$ with $y$ different from $a q^{-1}$ we have $\phi_{1} f\left(\frac{q y x}{a}, x\right)=\sum_{k=0}^{\infty} \sum_{s=0}^{\infty} \frac{a_{k+s+1} q^{-s(k+1)}}{a^{k+1}\left(q^{k+1}-1\right)} x^{k}\left\{\begin{array}{l}x \\ s\end{array}\right\}\left\{\begin{array}{l}y \\ k\end{array}\right\}$

Then we immediately have $\left|\phi_{1} f\left(\frac{a g x}{a}, x\right)\right| \leq \max _{k, s \geq 0}\left\{\left|\frac{a_{k+s+1}}{q^{k+1}-1}\right|\right\}$ for all 
$x, y$ in $V_{q}$ with $y \neq a q^{-1}$ and so we have $\left\|\phi_{1} f\right\|_{\infty} \leq \max _{k, s \geq 0}\left\{\left|\frac{a_{k+s+1}}{q^{k+1}-1}\right|\right\}$. If $\max _{k, s \geq 0}\left\{\left|\frac{a_{k+s+1}}{a^{k+1}-1}\right|\right\}=0$ it is clear that $\left\|\phi_{1} f\right\|_{\infty}=\max _{k, s \geq 0}\left\{\left|\frac{a_{k+s+1}}{a^{k+1}-1}\right|\right\}$. If $\max _{k, s \geq 0}\left\{\left|\frac{a_{k+s+1}}{q^{k+1}-1}\right|\right\}>0$, then put $I=\{(i, j) \in \mathbb{N} \times \mathbb{N}$ : $\left.\left|\frac{a_{j+i+1}}{q^{j+1}-1}\right|=\max _{k, s \geq 0}\left\{\left|\frac{a_{k+s+1}}{q^{k+1}-1}\right|\right\}\right\}$. Now let $S=\min \{i \in \mathbb{N}$ : there exists a $j \in \mathbb{N}$ such that $(i, j) \in I\}$ and $T=\min \{t \in \mathbb{N}$ : $(S, t) \in I\}$ then it is easy to see that $\left|\phi_{1} f\left({ }_{a} a q^{S} a q^{T}, a q^{S}\right)\right|=$ $\left|\frac{a_{T}+S+1}{a^{T+1}-1}\right|=\max _{k, s \geq 0}\left\{\left|\frac{a_{k+s+1}}{q^{k+1}-1}\right|\right\}$ and so we conclude $\left\|\phi_{1} f\right\|_{\infty}=$ $\max _{k, s \geq 0}\left\{\left|\frac{a_{k+s+1}}{q^{k+1}-1}\right|\right\}=\max _{n \geq 1}\left\{\left|a_{n}\left(\rho_{n}\right)^{-1}\right|\right\}$. Since $\|f\|_{1}=$ $\max \left\{\|f\|_{\infty},\left\|\phi_{1} f\right\|_{\infty}\right\}=\max _{\max }\left\{\max _{n \geq 0}\left\{\left|a_{n}\right|\right\}, \max _{n \geq 1}\left\{\left|a_{n}\left(\rho_{n}\right)^{-1}\right|\right\}\right\}$ and since $\left|\left(\rho_{n}\right)^{-1}\right| \geq 1$ for all $n$ we conclude that $\|f\|_{1}=$ $\max _{n \geq 0}\left\{\left|a_{n}\left(\rho_{n}\right)^{-1}\right|\right\}$. From this it follows that $\left\|\left\{\begin{array}{l}x \\ n\end{array}\right\}\right\|_{1}=\left|\left(\rho_{n}\right)^{-1}\right|$ so $\left\|\rho_{n}\left\{\begin{array}{l}x \\ n\end{array}\right\}\right\|_{1}=1$. Furthermore, $f(x)=\sum_{n=0}^{\infty} a_{n}\left\{\begin{array}{l}x \\ n\end{array}\right\}=\sum_{n=0}^{\infty} \frac{a_{n}}{\rho_{n}} \rho_{n}\left\{\begin{array}{l}x \\ n\end{array}\right\}$ with $\|f\|_{1}=\max _{n \geq 0}\left\{\left|a_{n}\left(\rho_{n}\right)^{-1}\right|\right\}=\max _{n \geq 0}\left\{\left\|\frac{a_{n}}{\rho_{n}} \rho_{n}\left\{\begin{array}{l}x \\ n\end{array}\right\}\right\|_{1}\right\}$ so the functions $\left(\rho_{n}\left\{\begin{array}{l}x \\ n\end{array}\right\}\right)$ form an orthonormal base for $C^{1}\left(V_{q} \rightarrow K\right)$. This finishes the proof.

\section{References}

[1] Y. Amice, Les Nombres p-adiques. Presses Universitaires de France, Paris, 1975 (Collection SUP, Le Mathématicien,14).

[2] J. Araujo and W. H. Schikhof, The Weierstrass-Stone Approximation Theorem for p-adic $C^{n}$-functions, Annales Mathématiques Blaise Pascal, Volume 1, No 1, Janvier 1994, p. 61-74.

[3] L. Gruson and M. van der Put, Banach Spaces, Table Ronde d' Analyse non-archimédienne (1972 Paris) , Bulletin de la Société Mathématique de France, Memoire 39-40,1974, p. 55 - 100.

[4] K. Mahler, An Interpolation Series for Continuous Functions of a p-adic Variable, Journal für reine und angewandte Mathematik, vol. 199,1958 , p. $23-34$.

[5] W. H. Schikhof, Ultrametric Calculus : An Introduction to p-adic Analysis, Cambridge University Press, 1984. 
[6] A.C.M. van Rooij, Non-Anchimedean Functional Analysis, Marcel Dekker, 1978 (Pure and Applied Mathematics, 51).

[7] A. Verdoodt, Jackson's Formula with Remainder in p-adic Analysis, Indagationes Mathematicae, N.S., 4 (3), p. 375-384, september 1993.

[8] A. Verdoodt, Normal Bases for Non-Archimedean Spaces of Continuous Functions, Publicacions Matemàtiques, vol. 37, 1993, p. 403-427.

[9] A. Verdoodt, Normal Bases for the Space of Continuous Functions defined on a Subset of $Z_{p}$, Publicacions Matemàtiques, vol 38, $\mathrm{nr} 2$, 1994, p. 371-380.

Vrije Universiteit Brussel, Recibido: 6 de Febrero de 1995 Faculty of Applied Sciences,

Pleinlaan 2,

B-1050 Brussels, Belgium. 